\title{
Four-tangle for pure states
}

\author{
S. Shelly Sharma* \\ Depto. de Física, Universidade Estadual de Londrina, Londrina 86051-990, PR Brazil \\ N. K. Sharmat \\ Depto. de Matemática, Universidade Estadual de Londrina, Londrina 86051-990 PR, Brazil
}

\begin{abstract}
An expression for four-tangle is obtained by examining the negativity fonts present in a four-way partial transpose under local unitary operations. An alternate derivation of three tangle is also given.
\end{abstract}

Entanglement is inherent to interacting quantum systems and its quantification is an important question in quantum mechanics. Besides that entanglement is a physical resource for quantum communication [1], and a key ingredient for quantum computation [2, 3]. Negativity [4] of global partial transpose is a widely used computable measure of free bipartite entanglement. Negativity is based on Peres-Horodecki NPT criterion [5, 6] and is known to be an entanglement monotone [7]. It was shown in refs. [8, 9, 10] that the global partial transpose of an $N$-qubit state may be written as a sum of $K$-way partial transposes. In mathematics, the positive definiteness of matrix is checked by applying Sylvester's criterion. Our approach is an application of Sylvester's criterion and Peres-Horodecki NPT criterion aimed at relating the intrinsic eigenvalues characterizing partial $K$-way transposes of a composite system state operator to relevant invariants under local unitaries. A well known measure of purely tripartite entanglement is residual tangle or three-tangle [11], obtained by using concurrence [12], which measures entanglement of bipartite mixed state. In this letter, we examine the negativity fonts in a three way partial transpose and present a derivation of three tangle. In addition, an expression for four-tangle is obtained by examining the behavior of negativity fonts present in a four-way partial transpose under local unitary operations.

Firstly, what is a negativity font? A general N-qubit pure state reads as

$$
\left|\Phi^{A B C \ldots \ldots .}\right\rangle=\sum_{i_{1} i_{2} \ldots i_{N}} a_{i_{1} i_{2} \ldots i_{N}}\left|i_{1} i_{2} \ldots i_{N}\right\rangle
$$

where $\left|i_{1} i_{2} \ldots i_{N}\right\rangle$ are the basis vectors spanning the $2^{N}$ dimensional Hilbert space. The coefficients $a_{i_{1} i_{2} \ldots i_{N}}$ are complex numbers with $i_{m}=0$ and 1 , where $m=1, \ldots, N$. Consider the general two qubit state,

$$
\left|\Phi^{A B}\right\rangle=a_{00}|00\rangle_{A B}+a_{10}|10\rangle_{A B}+a_{01}|01\rangle_{A B}+a_{11}|11\rangle_{A B}
$$

with associated density matrix $\rho^{A B}$ representing the state operator $\widehat{\rho}^{A B}=\left|\Phi^{A B}\right\rangle\left\langle\Phi^{A B}\right|$. The squared global negativity of four by four matrix $\left(\rho^{A B}\right)_{G}^{T_{A}}$ obtained by partially transposing the state of qubit $A$ in $\rho^{A B}$, is given by $\left(N_{G}^{A}\right)^{2}=4\left|\operatorname{det}\left[\begin{array}{ll}a_{00} & a_{01} \\ a_{10} & a_{11}\end{array}\right]\right|$. Negativity is invariant with respect to unitaries $\mathrm{U}^{A}$ and $\mathrm{U}^{B}$. The matrix $\nu^{00}=\left[\begin{array}{ll}a_{00} & a_{01} \\ a_{10} & a_{11}\end{array}\right]$ represents the single negativity font present in $\left(\rho^{A B}\right)^{T_{A}}$. If $\operatorname{det} \nu^{00}=0$ the state is separable.

Some definitions are in place here. To simplify the notation we denote the vector $\left|i_{1} i_{2} \ldots i_{N}\right\rangle$ by $\left|\prod_{m=1}^{N} i_{m}\right\rangle$ and write density operator for the state as

$$
\widehat{\rho}=\sum_{\substack{i_{1}-i_{N}, j_{1}-j_{N}}}\left\langle\prod_{m=1}^{N} i_{m}|\widehat{\rho}| \prod_{m=1}^{N} j_{m}\right\rangle\left|\prod_{m=1}^{N} i_{m}\right\rangle\left\langle\prod_{m=1}^{N} j_{m}\right| .
$$

The matrix elements of global partial transpose of $\widehat{\rho}$ with respect to qubit $p$ are related to matrix elements of state operator through

$$
\left\langle\prod_{m=1}^{n} i_{m}\left|\hat{\rho}_{G}^{T_{p}}\right| \prod_{m=1}^{n} j_{m}\right\rangle=\left\langle j_{p} \prod_{m=1, m \neq p}^{n} i_{m}|\widehat{\rho}| i_{p} \prod_{m=1, m \neq p}^{n} j_{m}\right\rangle .
$$

\footnotetext{
*Electronic address: shelly@uel.br

†Electronic address: nsharma@uel.br
} 
To construct $K$-way partial transposes [10], every matrix element $\left\langle\prod_{m=1}^{N} i_{m}|\widehat{\rho}| \prod_{m=1}^{N} j_{m}\right\rangle$ is labelled by a number $K=\sum_{m=1}^{N}\left(1-\delta_{i_{m}, j_{m}}\right)$, where $\delta_{i_{m}, j_{m}}=1$ for $i_{m}=j_{m}$, and $\delta_{i_{m}, j_{m}}=0$ for $i_{m} \neq j_{m}$. The $K$-way partial transpose $(K>2)$ of $\rho$ with respect to subsystem $p$ is obtained by selective transposition such that

$$
\begin{aligned}
\left\langle\prod_{m=1}^{N} i_{m}\left|\hat{\rho}_{K}^{T_{p}}\right| \prod_{m=1}^{N} j_{m}\right\rangle & =\left\langle j_{p} \prod_{m=1, m \neq p}^{N} i_{m}|\hat{\rho}| i_{p} \prod_{m=1, m \neq p}^{N} j_{m}\right\rangle, \\
& \text { if } \quad \sum_{m=1}^{N}\left(1-\delta_{i_{m}, j_{m}}\right)=K, \text { and } \delta_{i_{p}, j_{p}}=0
\end{aligned}
$$

and

$$
\begin{aligned}
\left\langle\prod_{m=1}^{N} i_{m}\left|\hat{\rho}_{K}^{T_{p}}\right| \prod_{m=1}^{N} j_{m}\right\rangle & =\left\langle\prod_{m=1}^{N} i_{m}|\widehat{\rho}| \prod_{m=1}^{N} j_{m}\right\rangle, \\
\text { if } \quad \sum_{m=1}^{N}\left(1-\delta_{i_{m}, j_{m}}\right) & \neq K .
\end{aligned}
$$

while

$$
\begin{aligned}
\left\langle\prod_{m=1}^{N} i_{m}\left|\widehat{\rho}_{2}^{T_{p}}\right| \prod_{m=1}^{N} j_{m}\right\rangle & =\left\langle j_{p} \prod_{m=1, m \neq p}^{N} i_{m}|\widehat{\rho}| i_{p} \prod_{m=1, m \neq p}^{N} j_{m}\right\rangle, \\
& \text { if } \sum_{m=1}^{N}\left(1-\delta_{i_{m}, j_{m}}\right)=1 \text { or } 2, \quad \text { and } \quad \delta_{i_{p}, j_{p}}=0
\end{aligned}
$$

and

$$
\begin{aligned}
\left\langle\prod_{m=1}^{N} i_{m}\left|\hat{\rho}_{2}^{T_{p}}\right| \prod_{m=1}^{N} j_{m}\right\rangle & =\left\langle\prod_{m=1}^{N} i_{m}|\widehat{\rho}| \prod_{m=1}^{N} j_{m}\right\rangle, \\
\text { if } \quad \sum_{m=1}^{N}\left(1-\delta_{i_{m}, j_{m}}\right) & \neq 1 \text { or } 2 .
\end{aligned}
$$

Peres PPT separability criterion [5] states that the partial transpose $\widehat{\rho}_{G}^{T_{p}}$ of a separable state is positive. Sylvester's criterion states that a Hermitian matrix is positive-definite if and only if the matrix and all of the leading principal minors are positive. Principal minors are formed by removing row-column pairs from the original Hermitian matrix. Peres separability criterion translates to examining the leading principal minors of $\hat{\rho}_{G}^{T_{p}}$ for positivity. Entanglement is detected by demonstrating that there exists at least one principal minor of $\hat{\rho}_{G}^{T_{p}}$ which is negative. Let us examine a four by four sub-matrix of $\widehat{\rho}_{G}^{T_{p}}$ in the space spanned by vectors $\left|i_{1} i_{2} \ldots i_{N}\right\rangle,\left|j_{1} j_{2} \ldots j_{N}\right\rangle,\left|i_{1} i_{2} \ldots j_{p} \ldots i_{N}\right\rangle$, and $\left|j_{1} j_{2} \ldots i_{p} \ldots j_{N}\right\rangle$. If $\widehat{\rho}$ is a pure state, the sub-matrix may have a negative eigenvalue $\lambda^{-}=-\left|a_{i_{1} i_{2} \ldots i_{N}} a_{j_{1} j_{2} \ldots j_{N}}-a_{i_{1} i_{2} \ldots j_{p} \ldots i_{N}} a_{j_{1} j_{2} \ldots i_{p} \ldots j_{N}}\right|$ as such is a possible negativity font in $\widehat{\rho}_{G}$. In analogy with the two qubit case, a typical negativity font present in $\hat{\rho}_{G}^{T_{p}}$ is represented by the matrix

$$
\nu=\left[\begin{array}{cc}
a_{i_{1} i_{2} \ldots i_{N}} & a_{j_{1} j_{2} \ldots i_{p} \ldots j_{N}} \\
a_{i_{1} i_{2} \ldots j_{p} \ldots i_{N}} & a_{j_{1} j_{2} \ldots j_{N}}
\end{array}\right] .
$$

By shuffling the basis any one of the possible four by four sub-matrices of the form $\nu$ in $\widehat{\rho}_{G}^{T_{p}}$ can become a leading principal minor of $\widehat{\rho}_{G}^{T_{p}}$. Sylvester's criterion in the context of Peres condition implies that all possible leading four by four sub-matrices $\widehat{\rho}_{G}^{T_{p}}$ are positive for a separable state. An entangled state has at least one negative four by four sub-matrix (a negativity font) in $\widehat{\rho}_{G}^{T_{p}}$. Negativity of a given font is invariant with respect to a unitary transformation on subsystem $p$. The Global negativity

$$
N_{G}^{p}=\left(\left\|\rho_{G}^{T_{p}}\right\|_{1}-1\right)
$$


arising due to all the negativity fonts present in $\widehat{\rho}_{G}^{T_{p}}$ measures the entanglement of subsystem $p$ with it's complement. Here $\|\widehat{\rho}\|_{1}$ is the trace norm of $\hat{\rho}$.

The $K$-way negativity calculated from $K$-way partial transpose of matrix $\rho$ with respect to subsystem $p$, is defined as $N_{K}^{p}=\left(\left\|\rho_{K}^{T_{p}}\right\|_{1}-1\right)$. Using the definition of trace norm and the fact that $\operatorname{tr}\left(\rho_{K}^{T_{p}}\right)=1$, we get $N_{K}^{p}=2 \sum_{i}\left|\lambda_{i}^{K-}\right|$, $\lambda_{i}^{K-}$ being the negative eigenvalues of matrix $\rho_{K}^{T_{p}}$. The $K$-way negativity $(2 \leq K \leq N)$, defined as the negativity of $K$-way partial transpose, is determined by the presence or absence of $K$-way quantum coherences in the composite system. By $K$-way coherences we mean the type of coherences present in a $K$-qubit GHZ- like state. The negativity $N_{K}^{p}$ is a measure of all possible types of entanglement attributed to $K$ - way coherences.

It is straight forward to verify that

$$
\widehat{\rho}_{G}^{T_{p}}=\sum_{K=2}^{N} \widehat{\rho}_{K}^{T_{p}}-(N-2) \widehat{\rho}
$$

By rewriting the global partial transpose as a sum of $K$-way partial transposes, the negativity fonts are distributed amongst $N-1$ partial transposes.

\section{THREE-TANGLE}

The state operator for a general three qubit pure state may be rewritten as

$$
\left|\Psi^{A B C}\right\rangle=\left|\Phi_{000}^{A B C}\right\rangle+\left|\Phi_{001}^{A B C}\right\rangle, \quad \widehat{\rho}^{A B C}=\left|\Psi^{A B C}\right\rangle\left\langle\Psi^{A B C}\right|
$$

where

$$
\begin{aligned}
& \left|\Phi_{000}^{A B C}\right\rangle=a_{000}|000\rangle+a_{111}|111\rangle+a_{100}|100\rangle+a_{011}|011\rangle, \\
& \left|\Phi_{001}^{A B C}\right\rangle=a_{001}|001\rangle+a_{110}|110\rangle+a_{101}|101\rangle+a_{010}|010\rangle .
\end{aligned}
$$

The global partially transposed matrix $\left(\rho^{A B C}\right)_{G}^{T_{A}}$ is related to three-way and two-way partial transposes obtained from $\rho^{A B C}$ by selective partial transposition as in Eqs. (5, 8) through

$$
\left(\rho^{A B C}\right)_{G}^{T_{A}}=\left(\rho^{A B C}\right)_{3}^{T_{A}}+\left(\rho^{A B C}\right)_{2}^{T_{A}}-\rho^{A B C}
$$

The three way partial transpose $\left(\rho^{A B C}\right)_{3}^{T_{A}}$ has two negativity fonts

$$
\nu^{000}=\left[\begin{array}{ll}
a_{000} & a_{011} \\
a_{100} & a_{111}
\end{array}\right], \quad \text { and } \nu^{001}=\left[\begin{array}{ll}
a_{001} & a_{010} \\
a_{101} & a_{110}
\end{array}\right] .
$$

The square of negativity of $\left(\rho_{000}^{A B C}\right)_{3}^{T_{A}}$ is found to be $4\left|\operatorname{det}\left(\nu^{000}\right)\right|$ and that of $\left(\rho_{001}^{A B C}\right)_{3}^{T_{A}}$ is $4\left|\operatorname{det}\left(\nu^{001}\right)\right|$. After a local unitary $U^{B}=\frac{1}{\sqrt{1+|x|^{2}}}\left[\begin{array}{cc}1 & -x^{*} \\ x & 1\end{array}\right]$, the state $\left|\Psi^{A B C}\right\rangle$ reads as $U^{B}\left|\Psi^{A B C}\right\rangle=\sum_{i_{1} i_{2} i_{3}} b_{i_{1} i_{2} i_{3}}\left|i_{1} i_{2} i_{3}\right\rangle$. Defining

$$
\begin{aligned}
P_{B_{0}}^{00} & =\operatorname{det}\left[\begin{array}{ll}
a_{000} & a_{001} \\
a_{100} & a_{101}
\end{array}\right], \quad P_{B_{1}}^{00}=\operatorname{det}\left[\begin{array}{ll}
a_{010} & a_{011} \\
a_{110} & a_{111}
\end{array}\right], \\
T^{000} & =\operatorname{det}\left[\begin{array}{ll}
a_{000} & a_{011} \\
a_{100} & a_{111}
\end{array}\right], \quad T^{001}=\operatorname{det}\left[\begin{array}{ll}
a_{001} & a_{010} \\
a_{101} & a_{110}
\end{array}\right],
\end{aligned}
$$

and using primed symbols for similar determinants after unitary transformation, we obtain

$$
\begin{aligned}
& \left(T^{000}\right)^{\prime}=\frac{1}{1+|x|^{2}}\left(T^{000}+|x|^{2} T^{001}-x^{*} P_{B_{1}}^{00}+x P_{B_{0}}^{00}\right), \\
& \left(T^{001}\right)^{\prime}=\frac{1}{1+|x|^{2}}\left(T^{001}+|x|^{2} T^{000}+x^{*} P_{B_{1}}^{00}-x P_{B_{0}}^{00}\right),
\end{aligned}
$$




$$
\begin{gathered}
\left(P_{B_{0}}^{00}\right)^{\prime}=\frac{1}{1+|x|^{2}}\left(P_{B_{0}}^{00}+\left(x^{*}\right)^{2} P_{B_{1}}^{00}+x^{*}\left(T^{001}-T^{000}\right)\right) \\
\left(P_{B_{1}}^{00}\right)^{\prime}=\frac{1}{1+|x|^{2}}\left(P_{B_{1}}^{00}+x^{2} P_{B_{0}}^{00}-x\left(T^{001}-T^{000}\right)\right) .
\end{gathered}
$$

It is easily verified that $\left(T^{001}-T^{000}\right), P_{B 1}^{00}$ and $P_{B 0}^{00}$ are invariant under local unitaries on qubits $A$ and $C$. Using the relations given in Eqs. (14]17]) we obtain the three qubit invariant $T=\left(T^{001}-T^{000}\right)^{2}-4\left(P_{B 1}^{00}\right)\left(P_{B 0}^{00}\right)$, which determines the three-tangle [11] through

$$
\tau_{3}=4\left|\left(T^{001}-T^{000}\right)^{2}-4 P_{B 1}^{00} P_{B 0}^{00}\right| .
$$

It is further noted that the product $T^{001} T^{000}=P_{C_{0}}^{00} P_{C_{1}}^{00}-P_{B 1}^{00} P_{B 0}^{00}$, therefore alternate form of the invariant is $\left|\left(T^{001}+T^{000}\right)^{2}-4 P_{C_{0}}^{00} P_{C_{1}}^{00}\right|$. Starting from negativity fonts in global partial transpose with respect to $B$ and $C$ will yield the same invariant.

\section{FOUR TANGLE - A MEASURE OF GENUINE FOUR QUBIT ENTANGLEMENT}

In the case of a general four qubit state

$$
\left|\Psi^{A B C D}\right\rangle=\sum_{i_{1} i_{2} i_{3} i_{4}} a_{i_{1} i_{2} i_{3} i_{4}}\left|i_{1} i_{2} i_{3} i_{4}\right\rangle,
$$

four posible negativity fonts in $\left(\rho^{A B C D}\right)_{4}^{T_{A}}$ are identified as

$$
\begin{array}{ll}
\nu^{0000}=\left[\begin{array}{ll}
a_{0000} & a_{0111} \\
a_{1000} & a_{1111}
\end{array}\right], \quad \nu^{0001}=\left[\begin{array}{ll}
a_{0001} & a_{0110} \\
a_{1001} & a_{1110}
\end{array}\right], \\
\nu^{0010}=\left[\begin{array}{ll}
a_{0010} & a_{0101} \\
a_{1010} & a_{1101}
\end{array}\right], & \nu^{0011}=\left[\begin{array}{ll}
a_{0011} & a_{0100} \\
a_{1011} & a_{1100}
\end{array}\right] .
\end{array}
$$

Here qubits $A$ and $B$ are chosen as leading qubits. A unitary on qubit $A$ does not change $\operatorname{det}\left(\nu^{00 i_{3} i_{4}}\right)$.

After applying unitary transformation to qubit $D$ we obtain

$$
U^{D}\left|\Psi^{A B C D}\right\rangle=\sum_{i_{1} i_{2} i_{3} i_{4}} d_{i_{1} i_{2} i_{3} i_{4}}\left|i_{1} i_{2} i_{3} i_{4}\right\rangle
$$

Defining

$$
F_{00 i_{3} i_{4}}=\operatorname{det}\left[\begin{array}{ll}
a_{00 i_{3} i_{4}} & a_{01 i_{3}+1 i_{4}+1} \\
a_{10 i_{3} i_{4}} & a_{11 i_{3}+1 i_{4}+1}
\end{array}\right], \quad T_{C_{i_{3}}}^{00 i_{4}}=\operatorname{det}\left[\begin{array}{ll}
a_{00 i_{3} i_{4}} & a_{01 i_{3} i_{4}+1} \\
a_{10 i_{3} i_{4}} & a_{11 i_{3} i_{4}+1}
\end{array}\right],
$$

and using primed symbols for the same quantities calculated from coefficients $d_{i_{1} i_{2} i_{3} i_{4}}$, we can verify that

$$
\left(F_{0001}^{\prime}-F_{0000}^{\prime}\right) \pm\left(F_{0010}^{\prime}-F_{0011}^{\prime}\right)=\left(F_{0001}-F_{0000}\right) \pm\left(F_{0010}-F_{0011}\right) .
$$

Now application of unitary $U^{C}=\frac{1}{\sqrt{1+|y|^{2}}}\left[\begin{array}{cc}1 & -y^{*} \\ y & 1\end{array}\right]$ yields the state $U^{C}\left|\Psi^{A B C D}\right\rangle=\sum_{i_{1} i_{2} i_{3} i_{4}} c_{i_{1} i_{2} i_{3} i_{4}}\left|i_{1} i_{2} i_{3} i_{4}\right\rangle$, such that

$$
\begin{aligned}
F_{0001}^{\prime \prime}-F_{0000}^{\prime \prime}= & \frac{1}{\sqrt{1+|y|^{2}}}\left[\left(F_{0001}-F_{0000}\right)+|y|^{2}\left(F_{0010}-F_{0011}\right)\right. \\
& \left.+y^{*}\left(T_{C_{1}}^{000}-T_{C_{1}}^{001}\right)-y\left(T_{C_{0}}^{000}-T_{C_{0}}^{001}\right)\right], \\
F_{0010}^{\prime \prime}-F_{0011}^{\prime \prime}= & \frac{1}{\sqrt{1+|y|^{2}}}\left[\left(F_{0010}-F_{0011}\right)+|y|^{2}\left(F_{0001}-F_{0000}\right)\right. \\
& \left.-y^{*}\left(T_{C_{1}}^{000}-T_{C_{1}}^{001}\right)+y\left(T_{C_{0}}^{000}-T_{C_{0}}^{001}\right)\right],
\end{aligned}
$$


yielding

$$
\left(F_{0001}^{\prime \prime}-F_{0000}^{\prime \prime}\right)+\left(F_{0010}^{\prime \prime}-F_{0011}^{\prime \prime}\right)=\left(F_{0001}-F_{0000}\right)+\left(F_{0010}-F_{0011}\right) .
$$

To verify how four-way negativity fonts transform under unitary $U^{B}=\frac{1}{\sqrt{1+|z|^{2}}}\left[\begin{array}{cc}1 & -z^{*} \\ z & 1\end{array}\right]$, we write $U^{B}\left|\Psi^{A B C D}\right\rangle=$ $\sum_{i_{1} i_{2} i_{3} i_{4}} b_{i_{1} i_{2} i_{3} i_{4}}\left|i_{1} i_{2} i_{3} i_{4}\right\rangle$. Defining the determinant of three-way negativity font for qubits $\mathrm{ACD}$ in $\left|\Psi^{A B C D}\right\rangle$ as

$$
T_{B_{i_{2}}}^{00 i_{4}}=\operatorname{det}\left[\begin{array}{ll}
a_{0 i_{2} 0 i_{4}} & a_{0 i_{2} 1 i_{4}+1} \\
a_{1 i_{2} 0 i_{4}} & a_{1 i_{2} 1 i_{4}+1}
\end{array}\right]
$$

it is found that

$$
\begin{aligned}
& F_{0000}^{\prime \prime \prime}=\frac{1}{\sqrt{1+|z|^{2}}}\left[F_{0000}+|z|^{2} F_{0011}-z^{*} T_{B_{1}}^{000}+z T_{B_{0}}^{000}\right], \\
& F_{0001}^{\prime \prime \prime}=\frac{1}{\sqrt{1+|z|^{2}}}\left[F_{0001}+|z|^{2} F_{0010}-z^{*} T_{B_{1}}^{001}+z T_{B_{0}}^{001}\right], \\
& F_{0011}^{\prime \prime \prime}=\frac{1}{\sqrt{1+|z|^{2}}}\left[F_{0011}+|z|^{2} F_{0000}+z^{*} T_{B_{1}}^{000}-z T_{B_{0}}^{000}\right] \\
& F_{0010}^{\prime \prime \prime}==\frac{1}{\sqrt{1+|z|^{2}}}\left[F_{0010}+|z|^{2} F_{0001}+z^{*} T_{B_{1}}^{001}-z T_{B_{0}}^{001}\right],
\end{aligned}
$$

resulting in

$$
\left(F_{0001}^{\prime \prime \prime}+F_{0010}^{\prime \prime \prime}\right) \pm\left(F_{0000}^{\prime \prime \prime}+F_{0011}^{\prime \prime \prime}\right)=\left(F_{0001}+F_{0010}\right) \pm\left(F_{0000}+F_{0011}\right) .
$$

Combining the results of Eqs. (21), (24), and (30) with the fact that a unitary on qubit $A$ does not change det $\left(\nu^{00 i_{3} i_{4}}\right)$ the four qubit invariant is found to be $F^{A B C D}=\left(F_{0001}-F_{0000}\right)+\left(F_{0010}-F_{0011}\right)$. The measure of four qubit entanglement purely due to four-way correlations is defined as

$$
\tau_{4}=4\left|\left[\left(F_{0001}-F_{0000}\right)+\left(F_{0010}-F_{0011}\right)\right]^{2}\right| .
$$

We choose to call it four tangle since it measures four qubit GHZ state like entanglement in the same sense as Wootters's three tangle does for three qubits.

To summarize, an expression for four-tangle, a collective property of four qubits, has been obtained by using simple mathematics. Four tangle can be calculated from the coefficients in a general four qubit state without calculating the canonical state. The approach presented can be recursively applied to obtain other invariants as well as meaningful invariants in larger systems. The method may also elucidate the redistribution of quantum correlations in mixed states and help in obtaining eventually the entanglement measures for such states.

[1] A. K. Ekert, Phys. Rev. Lett. 67, 6611991 ; J. Kempe, Phys. Rev. A 60, 910 (1999).

[2] R. Jozsa and N. Linden, Proc. R. Soc. London, Ser. A 459, 2001 (2003) .

[3] R. Orus and J. I. Latorre, Phys. Rev. A 69, 052308 (2004).

[4] K. Zyczkowski, P. Horodecki, A. Sanpera, and M. Lewenstein, Phys. Rev. A 58, 883 (1998).

[5] A. Peres, Phys. Rev. Lett. 77, 1413 (1996).

[6] M. Horodecki, P. Horodecki, and R. Horodecki, Phys. Lett. A 223, 8 (1996).

[7] G. Vidal and R. F. Werner, Phys. Rev. Vol. 65, 032314 (2002).

[8] S. S. Sharma and N. K. Sharma, Phys. Rev. A 76, 012326 (2007).

[9] S. S. Sharma and N. K. Sharma, Phys. Rev. A 78, 012113 (2008).

[10] S. S. Sharma and N. K. Sharma, Phys. Rev. A 79, 062323 (2009).

[11] V. Coffman, J. Kundu, and W. K. Wootters, Phys. Rev. A 61, 052306 (2000).

[12] W. K. Wootters, Phys. Rev. Lett. 80, 2245 (1998). 